\title{
Acoustic Characterization of a Vessel-on-a-Chip Microfluidic System for Ultrasound- Mediated Drug Delivery
}

\author{
Inés Beekers ${ }^{(}$, Student Member, IEEE, Tom van Rooij ${ }^{\circledR}$, Member, IEEE, Martin D. Verweij, Member, IEEE, \\ Michel Versluis, Member, IEEE, Nico de Jong, Member, IEEE,
}

Sebastiaan J. Trietsch, and Klazina Kooiman ${ }^{\circledR}$, Member, IEEE

\begin{abstract}
Ultrasound in the presence of gas-filled microbubbles can be used to enhance local uptake of drugs and genes. To study the drug delivery potential and its underlying physical and biological mechanisms, an in vitro vessel model should ideally include 3-D cell culture, perfusion flow, and membrane-free soft boundaries. Here, we propose an organ-on-a-chip microfluidic platform to study ultrasound-mediated drug delivery: the OrganoPlate. The acoustic propagation into the OrganoPlate was determined to assess the feasibility of controlled microbubble actuation, which is required to study the microbubble-cell interaction for drug delivery. The pressure field in the OrganoPlate was characterized non-invasively by studying experimentally the well-known response of microbubbles and by simulating the acoustic wave propagation in the system. Microbubble dynamics in the OrganoPlate were recorded with the Brandaris 128 ultrahigh-speed camera (17 million frames/s) and a control experiment was performed in an OptiCell, an in vitro monolayer cell culture chamber that is conventionally used to study ultrasound-mediated drug delivery. When insonified at frequencies between 1 and $2 \mathrm{MHz}$, microbubbles in the OrganoPlate experienced larger oscillation amplitudes resulting from higher local pressures. Microbubbles responded similarly in both systems when insonified at frequencies between 2 and $4 \mathrm{MHz}$. Numerical simulations performed with a 3-D finite-element model of ultrasound propagation into the OrganoPlate and the OptiCell showed the same frequency-dependent behavior. The predictable and homogeneous pressure field in the OrganoPlate demonstrates its potential to develop an in vitro 3-D cell culture model, well suited to study ultrasound-mediated drug delivery.
\end{abstract}

Manuscript received October 15, 2017; accepted February 3, 2018. Date of publication February 7, 2018; date of current version March 30, 2018 This work was supported in part by the Applied and Engineering Sciences TTW (Veni-project 13669), a part of NWO, and in part by NanoNextNL, a micro- and nanotechnology consortium of the Government of The Netherlands and 130 partners. (Corresponding author: Inés Beekers.)

I. Beekers, T. van Rooij, and K. Kooiman are with the Department of Biomedical Engineering, Thorax Center, Erasmus MC, 3000 CA Rotterdam, The Netherlands (e-mail: d.beekers@erasmusmc.nl; k.kooiman@erasmusmc.nl).

M. D. Verweij and N. de Jong are with the Department of Biomedical Engineering, Thorax Center, Erasmus MC, 3000 CA Rotterdam, The Netherlands, and also with the Laboratory of Acoustical Wavefield Imaging, Delft University of Technology, 2628 CD Delft, The Netherlands.

M. Versluis is with the Physics of Fluids Group, MIRA Institute for Biomedical Technology and Technical Medicine and MESA+ Institute for Nanotechnology, University of Twente, 7522 NB Enschede, The Netherlands.

S. J. Trietsch is with Mimetas B.V.-The Organ-on-a-Chip Company, 2333 CH Leiden, The Netherlands (e-mail: s.trietsch@mimetas.com).

This paper has supplementary downloadable material available at http://ieeexplore.ieee.org, provided by the author. The file consists of PZFlex software implementation of acoustic propagation in the OrganoPlate and OptiCell.

Digital Object Identifier 10.1109/TUFFC.2018.2803137
Index Terms-Acoustic characterization, acoustic wave modeling, drug delivery, microfluidics, organ-on-a-chip, ultrasound contrast agents.

\section{INTRODUCTION}

$\mathbf{U}$ LTRASOUND contrast agents consist of coated gas microbubbles with diameters ranging from 1 to $10 \mu \mathrm{m}$ and are widely used to improve the contrast of organ perfusion in diagnostic ultrasound imaging. Upon ultrasound insonification, microbubbles compress and expand due to the acoustic pressure wave. This oscillatory behavior is the characteristic microbubble response which provides contrast enhancement for imaging. Recent studies also demonstrate the potential of oscillating microbubbles to locally enhance vascular drug delivery [1], [2]. Although the exact mechanism of delivery is unknown at present, there are three known pathways for ultrasound-mediated drug delivery [1]: 1) formation of pores in the cell membrane, termed sonoporation [2], [3]; 2) stimulation of endocytosis [4]; and 3) opening of cell-cell junctions into the extravascular tissue [5]. To study these pathways and elucidate the mechanisms, the in vitro endothelial cell model ideally includes 3-D cell culture, perfusion flow, and soft boundaries in the absence of rigid membranes. Since microbubble behavior strongly depends on the underlying substrate [6]-[8], the soft boundaries of an in vivo blood vessel need to be optimally reproduced. To achieve physiologically relevant cell behavior, 3-D cell culture and flow are required to mimic microcirculation, lumen architecture, and spatial distribution [9]-[11].

Ultrasound-mediated drug delivery studies commonly use an OptiCell (Nunc, Thermo Fisher Scientific, Wiesbaden, Germany) [12]-[18]. This parallel plate chamber limits cell culture to conventional monolayers on rigid boundaries and static conditions. In order to incorporate flow, others have used a commercially available microchannel flow setup ( $\mu$-Slide, Ibidi GmbH, Munich, Germany) [19], [20]. In addition, a biologically and acoustically compatible device was developed by Carugo et al. [21] for monolayer cell culture under flow. So far, these in vitro models may include physiologically relevant flow, but are unfortunately still limited to cell monolayers and rigid boundaries. Vessel-on-a-chip cell culture in a microfluidic device better reproduces the in vivo physical architecture of a vessel [22]. Ideally, a high throughput is desired, as well as cell 

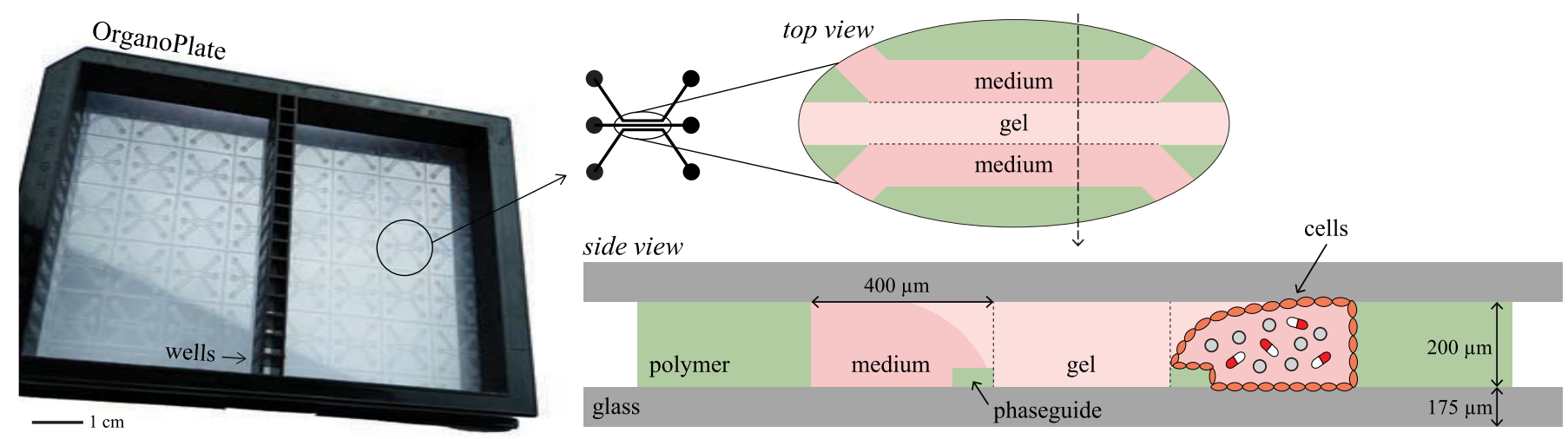

Fig. 1. Modified OrganoPlate, revealing the microfluidic structure after removing most of the wells (black plastic) of the standard 384-well microtiter plate. The scale bar indicates the dimensions of the picture. The top view shows the three adjacent microchannels: two for medium perfusion and one for gel (i.e., extracellular matrix), allowing for culture against soft boundaries. The arrow indicates the direction of the side view. The side view shows the microchannels encased between two glass plates (gray), bound by polymer walls (green), and separated by polymer phaseguides (green). The meniscus-pinning effect [24] caused by the phaseguides results in a curved gel as soft boundary. The side view also illustrates the desired 3-D in vitro setup with cultured endothelial cells (orange) which can be perfused with microbubbles (gray spheres) and therapeutic agent (red/white). (Not drawn to scale.)

culture biocompatible materials, a standarized manufacturing process, and optical and chemical access to both the apical and basolateral side of the vessel. To add functionality for ultrasound-mediated drug delivery, we propose to use the OrganoPlate ${ }^{\circledR}$ [23] (Mimetas B.V., Leiden, The Netherlands) to study the drug delivery pathways enhanced by microbubbles upon ultrasound insonification.

The OrganoPlate is an organ-on-a-chip platform with up to 96 microfluidic channel networks incorporated into a standard 384-well microtiter plate (Fig. 1). The 4003400B OrganoPlate used here contains 40 microfluidic networks, consisting of three adjacent microchannels allowing for 3-D cell culture, perfusion, soft boundaries, and co-culture. The adjacent microchannels are separated by phaseguides: capillary pressure barriers that are used to guide fluid flow and pattern extracellular matrices. The diffusion distances are shortened through the use of capillary pressure barriers instead of membranes or walls and allow for direct cellcell interactions [24]. Intricate cell models can be developed in the OrganoPlate; such as 3-D neuronal networks [25], intestinal epithelium tubes [26], and a functional hepatocyte liver model [27]. In this paper, we propose to use the OrganoPlate in combination with ultrasound for the first time. Since the microchannels are incorporated between two glass plates and separated by polymer walls, here we investigate how ultrasound propagates into the OrganoPlate.

The aim of this paper was to assess whether controlled microbubble oscillation is feasible in the OrganoPlate. Since microbubble response is dictated directly by the ultrasound pressure wave, the acoustic propagation into the OrganoPlate was characterized. Because of the small dimensions of the microchannels, the pressure field inside the OrganoPlate could not be measured using a hydrophone without altering the ultrasound field. To characterize the pressure in situ and noninvasively, microbubbles were used as pressure sensors. The pressure was determined from the well-known microbubble response upon insonification, which requires characterization of the microbubble shell parameters. Since these microbubble shell parameters depend on the pressure itself [28], [29], a new iterative method was introduced that both determined the pressure field to which the microbubbles were exposed and their corresponding shell parameters. In order to do so, individual microbubbles in the OrganoPlate were recorded during ultrasound insonification using the Brandaris 128 ultrahigh-speed camera [30]. Microbubble spectroscopy [31] was performed by successively insonifying each individual microbubble while sweeping through a range of transmit frequencies $f_{T}$ from 1 to $4 \mathrm{MHz}$. To characterize the ultrasound propagation, the pressure amplitude experienced by the microbubbles inside the OrganoPlate was obtained from the experimental data. The results were compared to those of control experiments performed in a conventional OptiCell, using an identical experimental setup [32]. In addition, a 3-D finite-element model was developed to simulate the acoustic propagation, to study the spatial distribution of the pressure field and to compare it with the experimental data.

\section{Material and Methods}

\section{A. OrganoPlate}

The OrganoPlate (Mimetas B.V., 4003400B) is a microfluidic system consisting of 40 chips developed for 3-D cell culture [23]. In the case of a three-lane design, each chip consists of three adjacent microchannels that can be accessed through their corresponding in- and outlets. The adjacent microchannels are separated by phaseguides [24], which are $100 \mu \mathrm{m} \times 50 \mu \mathrm{m}(w \times h)$ in size. As illustrated in Fig. 1, cells cultured adjacent to a microchannel with extracellular matrix can grow into a 3-D perfusable microvessel. The $400 \mu \mathrm{m} \times 200 \mu \mathrm{m} \times 2200 \mu \mathrm{m}(w \times h \times l)$ microchannels are incorporated between two $175-\mu \mathrm{m}$-thick borosilicate glass plates (Fig. 1). The three microchannels are bound between two polymer walls (400 $\mu \mathrm{m}$ wide; Fig. 1). The OrganoPlate was modified to ensure compatibility with our experimental setup. The standard wells of the 384-well microtiter plate were cut out, leaving only a single column of wells in the 


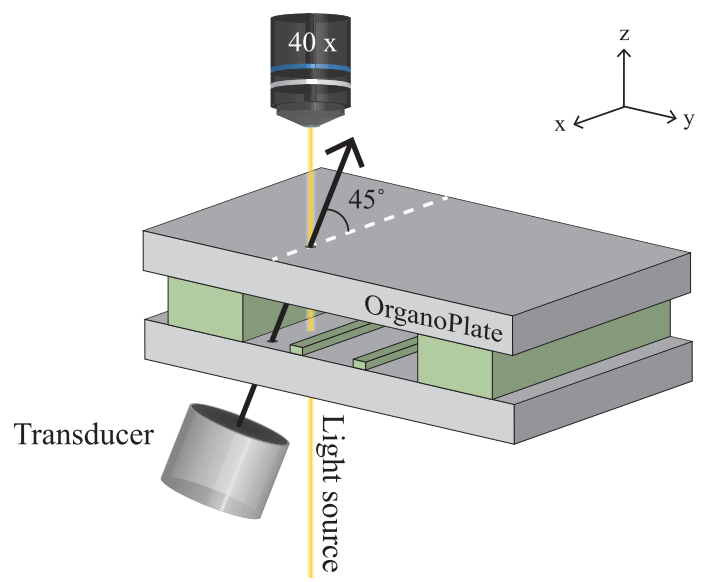

Fig. 2. Schematic of the experimental setup to study microbubble oscillation upon ultrasound insonification under a $45^{\circ}$ incidence angle. The optical and ultrasound foci are located just beneath the upper glass plate of the OrganoPlate. The entire setup was submersed in water. (Not drawn to scale.)

middle of the OrganoPlate, as depicted in Fig. 1. Removing the wells allowed for the visualization of the microchannels using an upright microscope and insonification from below at a $45^{\circ}$ incidence angle while submersed in water, thereby minimizing the reflections of the incident ultrasound wave with the microscope objective, as schematically depicted in Fig. 2.

\section{B. Microbubble Preparation}

Biotinylated lipid-coated microbubbles with a $\mathrm{C}_{4} \mathrm{~F}_{10}$ gas core and a 1,2-distearoyl-sn-glycero-3-phosphocholine (DSPC)-based shell were made by sonication for $10 \mathrm{~s}$, as previously described [32]-[34]. The microbubbles were passively washed by flotation in a $3 \mathrm{~mL}$ syringe with a one-way tap by leaving them to stand on the lab bench. After $45 \mathrm{~min}$, the subnatant was drained through the one-way tap and the microbubbles were resuspended in $1 \mathrm{~mL}$ of phosphate buffered saline (PBS) saturated with $\mathrm{C}_{4} \mathrm{~F}_{10}$ and drained into an Eppendorf tube. The microbubbles were diluted to a concentration of approximately $3 \times 10^{6}$ microbubbles $/ \mathrm{mL}$ in PBS as determined by a Coulter counter Multisizer $3(n=3)$ (Beckman Coulter, Mijdrecht, The Netherlands) using a $50 \mu \mathrm{m}$ aperture tube, allowing for the quantification of microbubble diameters of $1-30 \mu \mathrm{m}$.

\section{Experimental Setup}

Microbubble oscillations were studied in the OrganoPlate replicating the ultrasound settings by van Rooij et al. [32]. Briefly, a single-element broadband transducer (1-9 $\mathrm{MHz}$ bandwidth, 25-mm focal distance, $-6 \mathrm{~dB}$ beamwidth at $1 \mathrm{MHz}$ of $1.3 \mathrm{~mm}$, PA275, Precision Acoustics, Dorchester, U.K.) was used for insonification at a $45^{\circ}$ incidence angle while submersed in water (Fig. 2). The transducer output was calibrated in a separate experiment using a needle hydrophone (1-mm diameter, PA2293, Precision Acoustics). To perform microbubble spectroscopy measurements, we insonified single microbubbles with transmit frequencies $\left(f_{T}\right)$ ranging from 1 to $4 \mathrm{MHz}$, in steps of $300 \mathrm{kHz}$, using an eight-cycle sine wave burst. The first and last cycles were Gaussian tapered (variance $\sigma^{2}=1.1 / f_{T}^{2}$ ). Measurements were performed at 20-kPa peak negative pressure (PNP) in the focus, as calibrated in water. The optical and ultrasound foci were aligned and situated just below the upper glass plate of the OrganoPlate, where the microbubbles were located.

In this study, microbubbles were introduced into the OrganoPlate by pipetting $6 \mu \mathrm{L}$ of microbubble suspension on the inlet of a microchannel. They were insonified at room temperature within $2 \mathrm{~h}$. Their oscillation behavior was recorded using the Brandaris 128 ultrahigh-speed camera at approximately 17 million frames/s [30], using a microscope (BX-FM, Olympus, Tokyo, Japan) with a $40 \times$ water immersion objective (LUMPlanFl, Olympus) and a $2 \times$ lens (Olympus). The first recording was performed in the absence of ultrasound to image the initial microbubble size, followed by 11 recordings with ultrasound sweeping through the different transmit frequencies. The time between recordings was $80 \mathrm{~ms}$. Recorded microbubbles were separated at least $0.7 \mathrm{~mm}$ to avoid multiple insonifications at the same location due to the finite $-6 \mathrm{~dB}$ beamwidth of the transducer. In total, data from 12 different microchannels were evaluated.

\section{Experimental Data Analysis}

Microbubble oscillations were quantified using customdesigned image analysis software to determine the change in microbubble radius as a function of time ( $R-t$ curve) [31]. The relative excursion $x(t)$ was determined as $R=R_{0}(1+x)$, with $R_{0}$ being the resting radius. To study the oscillations at the transmit frequency a third-order Butterworth bandpass filter was applied to $x(t)$, centered at $f_{T}$ with a $500-\mathrm{kHz}$ bandwidth. The maximum amplitude of the filtered signal was defined as the relative excursion amplitude $x_{0}\left(f_{T}\right)$.

The relationship between the relative excursion amplitude $x_{0}\left(f_{T}\right)$ and the acoustic pressure amplitude experienced by the microbubble $P$ was determined. In principle, microbubbles insonified at low enough acoustic pressures behave as linear oscillators [35], [36]. Therefore, the amplitude $x_{0}$ of the relative excursion $x=x_{0} \sin \left(\omega_{T} t\right)$ can be described by the expression for a harmonic oscillator [31]

$$
x_{0}=\frac{F_{0}}{\sqrt{\left(\omega_{0}^{2}-\omega_{T}^{2}\right)^{2}+\left(\delta \omega_{T} \omega_{0}\right)^{2}}}
$$

with $\omega_{0}=2 \pi f_{0}$, where $f_{0}$ is the eigenfrequency of the system, $\omega_{T}=2 \pi f_{T}$, with $f_{T}$ being the transmit frequency that drives the oscillation, and $\delta$ is the total damping coefficient. $F_{0}$ is the amplitude of the forcing term $F(t)=F_{0} \sin \left(\omega_{T} t\right)$ and is given by $F_{0}=|P| /\left(\rho R_{0}^{2}\right)[1]$, with $\rho=10^{3} \mathrm{~kg} / \mathrm{m}^{3}$ being the density of water. The eigenfrequency $f_{0}$ of the system is

$$
f_{0}=\frac{1}{2 \pi} \sqrt{\frac{1}{\rho R_{0}^{2}}\left[3 \gamma P_{0}+\frac{2(3 \gamma-1) \sigma_{w}}{R_{0}}+\frac{4 \chi}{R_{0}}\right]}
$$

with $\gamma=1.07$ being the ratio of specific heats for $\mathrm{C}_{4} \mathrm{~F}_{10}$, $P_{0}=10^{5} \mathrm{~Pa}$ being the ambient pressure, $\sigma_{w}=0.072 \mathrm{~N} / \mathrm{m}$ being the surface tension, and $\chi$ being the elasticity of the microbubble shell as obtained from the linearized microbubble dynamics equation [31]. The total damping coefficient includes 


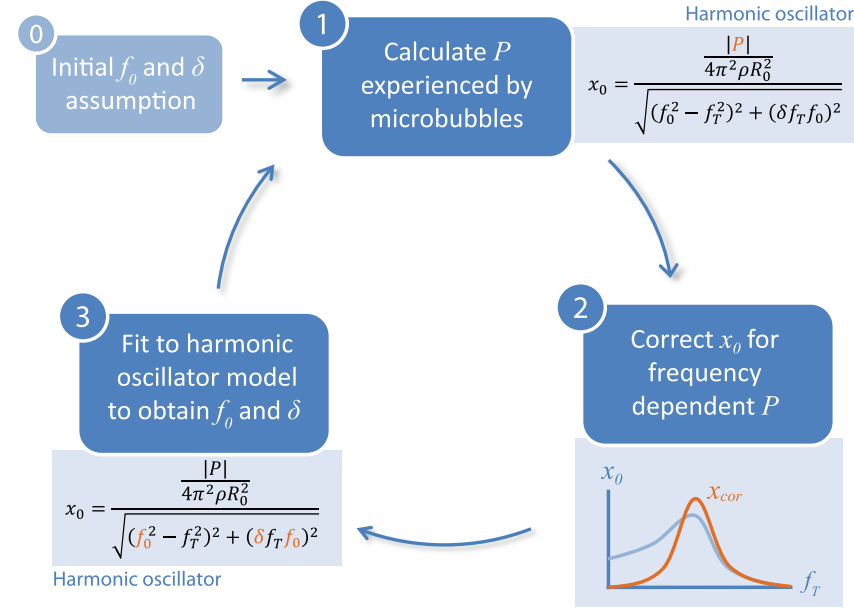

Fig. 3. Schematic of the iterative method to fit the relative excursion amplitude $\left(x_{0}\right)$ to the harmonic oscillator model (4) to determine the pressure amplitude $(P)$ and the microbubble shell parameters, incorporated in the eigenfrequency $\left(f_{0}\right)$ and damping coefficient $(\delta)$. Step 1 is repeated for each transmit frequency $\left(f_{T}\right)$ and steps 2 and 3 for each microbubble. The parameters determined in each step are shown in orange.

the sound reradiated by the microbubble, a contribution by liquid viscosity, a thermal contribution assumed equal to the viscous contribution [35], and the effect of the shell viscosity [32]

$$
\delta=\frac{\omega_{0} R_{0}}{c}+2 \cdot \frac{4 \mu}{R_{0}^{2} \rho \omega_{0}}+\frac{4 \kappa_{s}}{R_{0}^{3} \rho \omega_{0}}
$$

with $c=1500 \mathrm{~m} / \mathrm{s}$ being the speed of sound in water, $\mu=10^{-3} \mathrm{~Pa} \cdot \mathrm{s}$ being the viscosity of water, and $\kappa_{s}$ being the microbubble shell viscosity. Finally, the relative excursion amplitude $x_{0}$ in (1) can be rewritten as a function of the acoustic pressure amplitude and transmit frequency

$$
x_{0}=\frac{|P| /\left(4 \pi^{2} \rho R_{0}^{2}\right)}{\sqrt{\left(f_{0}^{2}-f_{T}^{2}\right)^{2}+\left(\delta f_{T} f_{0}\right)^{2}}}
$$

where $f_{0}$ and $\delta$ depend on the microbubble shell as given in (2) and (3). Hence, in the linear regime, we can predict the relative excursion amplitude of a microbubble if we know the pressure amplitude, the transmit frequency, and the microbubble's resting size, shell elasticity, and shell viscosity. Equivalently, if we know $x_{0}$ and $R_{0}$ we can determine the pressure experienced by the microbubble from the experimental data, either in the OrganoPlate or in the OptiCell, when the shell elasticity and viscosity are known.

The shell properties of DSPC-coated microbubbles were determined by van Rooij et al. [32] by fitting microbubble excursion to the response of a linear oscillator, at $20-\mathrm{kPa}$ PNP in an OptiCell. However, to account for the variability among microbubble size, shell properties, and the change in shell elasticity with pressure [28], [29], shell characterization was incorporated in our analysis with a new iterative method consisting of three steps, as described and visualized in Fig. 3.

Step 0 - Initial conditions: The initial conditions for the eigenfrequency $\left(f_{0}\right)$ and damping $(\delta)$ of each microbubble were chosen by assuming the general shell properties by van Rooij et al. [32]. For each microbubble, $f_{0}$ was determined using (2), the corresponding $R_{0}$, and $\chi=0.26 \mathrm{~N} / \mathrm{m}$ [32]. Using (3), $\delta$ was determined with the $R_{0}$ of each microbubble and a logarithmic fit through the relationship between viscosity and microbubble radius of $\log _{10}\left(\kappa_{S}\right)=0.188 \cdot R_{0}-8.838$ [32].

Step 1 - Calculate $P\left(f_{T}\right)$ experienced by microbubbles: With the initial conditions $R_{0}$ and the known relative excursion amplitudes $x_{0}\left(f_{T}\right)$, the pressure amplitude experienced by each microbubble was calculated according to the harmonic oscillator model (4) (Step 1 in Fig. 3). This first step was repeated for every $f_{T}$ from 1 to $4 \mathrm{MHz}$ and resulted in a frequency-dependent pressure $P\left(f_{T}\right)$ experienced by the microbubbles. To characterize the acoustic propagation into the system, the pressure inside the microchannel was normalized to the applied pressure, $P^{*}=M(P) / P_{A}$, with $M(P)$ the median $P$ experienced by microbubbles when insonified at $f_{T}$ and the applied pressure $P_{A}(20-\mathrm{kPa} \mathrm{PNP})$. When $P^{*}$ is represented in decibel $(\mathrm{dB})$, it is defined as $20 \cdot \log _{10}\left(P^{*}\right)$.

Step 2 - Correct $x_{0}$ for the frequency dependent $P\left(f_{T}\right)$ : In order to determine the microbubble shell parameters a standard spectroscopy data set is required, consisting of $x_{0}$ as a function of $f_{T}$ for each microbubble while insonified at uniform pressure amplitude [31]. However, since the pressure in the microchannels found in Step 1 is frequency dependent, $P^{*}\left(f_{T}\right)$, the microbubbles experienced a different pressure at different $f_{T}$. We therefore corrected the relative excursion amplitude for the frequency-dependent pressure, $x_{\text {cor }}=x_{0} / P^{*}\left(f_{T}\right)$ (Step 2 of Fig. 3).

Step 3 - Fit to harmonic oscillator to obtain shell properties: The corrected relative excursion amplitude $x_{\text {cor }}$ was fit to the model of a harmonic oscillator (4) by a least-mean-squares method to determine $f_{0}$ and $\delta$ for every microbubble (Step 3 in Fig. 3). This third step resulted in a specific $f_{0}$ and $\delta$ for each individual microbubble, accounting for the variability of shell properties among them.

Using the specific properties of each individual microbubble, the pressure amplitude experienced by each microbubble was recalculated, returning to the first step of the iterative scheme (Step 1 in Fig. 3). In total, 30 iterations were carried out to stabilize the obtained $P^{*}, f_{0}$, and $\delta$. The same data analysis was also performed on the $R-t$ curves obtained by van Rooij et al. [32] in an OptiCell ( $n=30$ microbubbles). All analyses were performed using MATLAB (The Mathworks Inc., Natick, MA, USA).

\section{E. Finite-Element Model}

A 3-D wave propagation model was set up in PZFlex (PZFlex LLC, Cupertino, CA, USA), a time-domain finiteelement package. Three different models were simulated: the OrganoPlate, the OptiCell, and the free field in water. The models are shown in Fig. 4 and the software implementation can be found in the Supplementary Material.

The model of the OrganoPlate included the glass plates, polymer walls, and phaseguides separating the microchannels, as shown in Fig. 4(a). The acoustic material properties of PMMA were used to approximate the properties of the 
a) OrganoPlate

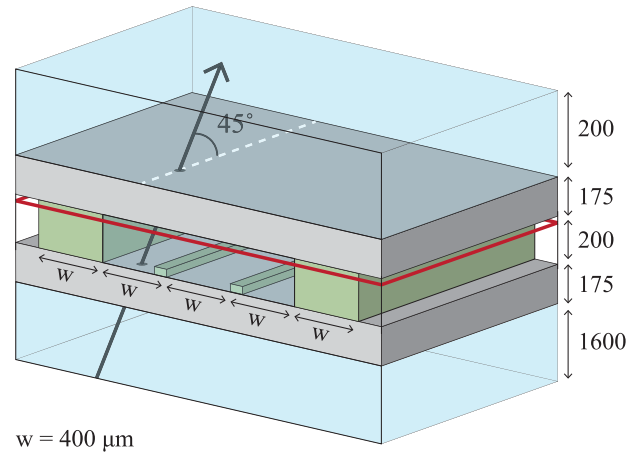

Water $\square$ Glass $\square$ PMMA $\square$ Air b) OptiCell

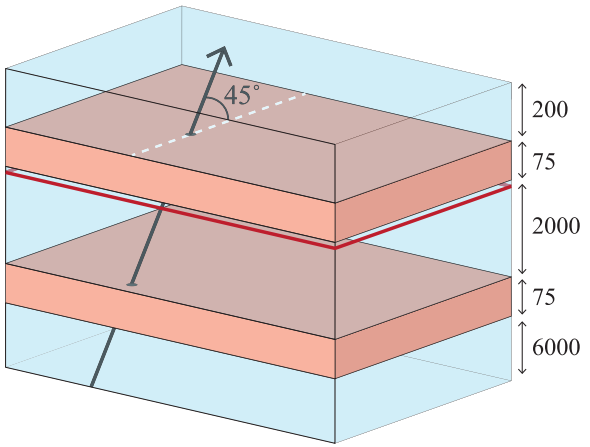

$\square$ Water $\square$ Polystyrene c) Free field

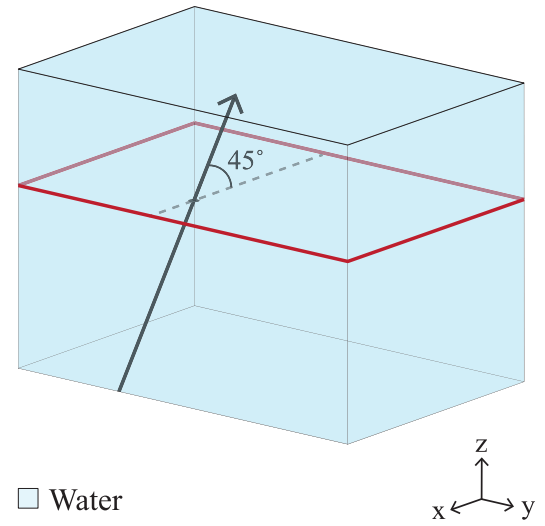

Fig. 4. Schematics of the 3-D PZFlex models (not drawn to scale) and the ultrasound insonification under a $45^{\circ}$ incidence angle. The plane delineated in red corresponds to the $x y$-plane at which the ultrasound beam was focused and the pressure $\left(P_{\text {sim }}\right)$ was evaluated. The dimensions are indicated in $\mu \mathrm{m}$. (a) Model of the OrganoPlate with total dimensions of $4200 \mu \mathrm{m} \times 2800 \mu \mathrm{m} \times 2400 \mu \mathrm{m}(x \times y \times z)$. The phaseguides are $4200 \mu \mathrm{m} \times 100 \mu \mathrm{m} \times 50 \mu \mathrm{m}$ $(x \times y \times z)$. The microchannels were filled with water and the outer spacing with air. (b) Model of the OptiCell with total dimensions of $12000 \mu \mathrm{m} \times$ $8100 \mu \mathrm{m} \times 8400 \mu \mathrm{m}(x \times y \times z)$. (c) Model of the free field (water), which was simulated for both the dimensions of (a) and (b).

TABLE I

Acoustic Material Properties Compiled From Literature: Borosilicate GLASS [37], [38], PMMA [39], AND POLYSTYREne [40]

\begin{tabular}{|rccc|}
\hline & Borosilicate glass & PMMA & Polystyrene \\
\hline Density $\left(\mathrm{g} \mathrm{cm}^{-3}\right)$ & 2.51 & 1.20 & 1.05 \\
\hline Longitudinal velocity $\left(\mathrm{m} \mathrm{s}^{-1}\right)$ & 5710 & 2757 & 2400 \\
\hline $\begin{array}{r}\text { Shear velocity }\left(\mathrm{m} \mathrm{s}^{-1}\right) \\
\text { Longitudinal attenuation } \\
\left(\mathrm{dB} \mathrm{cm}^{-1} \mathrm{MHz}^{-1}\right)\end{array}$ & 3467 & 1400 & 1150 \\
$\begin{array}{r}\text { Shear attenuation } \\
\left(\mathrm{dB} \mathrm{cm}^{-1} \mathrm{MHz}^{-1}\right)\end{array}$ & 0.8 & 1.64 & 0.35 \\
\hline
\end{tabular}

polymer walls and phaseguides. The acoustic material properties of borosilicate glass and PMMA are given in Table I. To replicate the insonification conditions of the experimental setup, the entire OrganoPlate was submersed in water and the three adjacent lanes of the microfluidic network were filled with water. The ultrasound pressure wave originated from a curved disk with the same ratio of curvature radius to diameter as the PA275 transducer (25-mm curvature radius and 23-mm diameter). A sine wave burst of eight cycles and unit amplitude was emitted from below at an incidence angle of $45^{\circ}$ (Fig. 4). The transmit frequency was varied between 1 and $4 \mathrm{MHz}$. The geometrical focus was located in the middle of the medium channel just beneath the surface of the glass plate, corresponding to the location of the microbubbles during the experiment. The grid size was at least 15 elements per wavelength. The OptiCell was modeled by two polystyrene (Table I) parallel plates, each with a thickness of $75 \mu \mathrm{m}$ and separated by $2 \mathrm{~mm}$ [17] [see Fig. 4(b)]. The same insonification setup was simulated for the OptiCell, but now with the ultrasound focus located just below the upper polystyrene plate. To assess the acoustic transparency of both the OrganoPlate and the OptiCell, the wave propagation in the free field was evaluated in the same grid and for the same ultrasound settings [Fig. 4(c)]. For all simulations, both the longitudinal and shear wave components of the pressure wave propagation were calculated.

The finite-element model provides time-domain information on the pressure wave propagating through each element. The simulated pressure amplitude $\left(P_{\text {sim }}\right)$ was defined as the median of the rectangular windowed envelope of the time varying pressure and evaluated in the $x y$-plane just below the upper glass plate or the polystyrene membrane (Fig. 4). We defined $P_{\text {sim }}^{*}=20 \log _{10}\left(P_{\text {sim }} / P_{\text {ref }}\right)$, normalizing $P_{\text {sim }}$ with respect to the maximum pressure amplitude obtained in the free field $\left(P_{\text {ref }}\right)$, in other words, at the focus when transmitted at the same frequency in water. In the interest of comparing the modeled pressure wave propagation to the experimental data, the median and interquartile range (IQR) of $P_{\mathrm{sim}}^{*}$ were determined in a region equivalent to the field of view around the focus $(100 \mu \mathrm{m} \times 100 \mu \mathrm{m})$.

\section{RESULTS}

\section{A. Microbubble Oscillation Behavior}

Single microbubbles with $R_{0}$ from 1.4 to $3.1 \mu \mathrm{m}$ were insonified and recorded in the OrganoPlate $(n=29)$. The size range was similar to that previously recorded in the OptiCell $(n=30)$ [32]. A selection of typical frames of a recorded microbubble in the OrganoPlate are shown in Fig. 5, for $f_{T}=1.6 \mathrm{MHz}$. Examples of the determined $x_{0}$ as a function of $R_{0}$ are shown for $f_{T}=1 \mathrm{MHz}$ [Fig. 6(a)], $1.6 \mathrm{MHz}$ [Fig. 6(b)], and $2.8 \mathrm{MHz}$ [Fig. 6(c)]. Although variations in response between microbubbles of similar size were observed in the spread of the data points, at $f_{T}=1$ and $1.6 \mathrm{MHz}$ the typical $x_{0}$ in the OrganoPlate was larger than that in the OptiCell. On the other hand, at $f_{T}=2.8 \mathrm{MHz}, x_{0}$ was similar in both the OrganoPlate and the OptiCell.

By iteratively fitting $x_{0}$ to the harmonic oscillator model, the microbubble shell parameters and $P$ experienced by the microbubbles were obtained. The normalized median pressure $\left(P^{*}\right)$ experienced by the microbubbles is shown in Fig. 7. The change of $P^{*}$ for increasing number of iterations at 

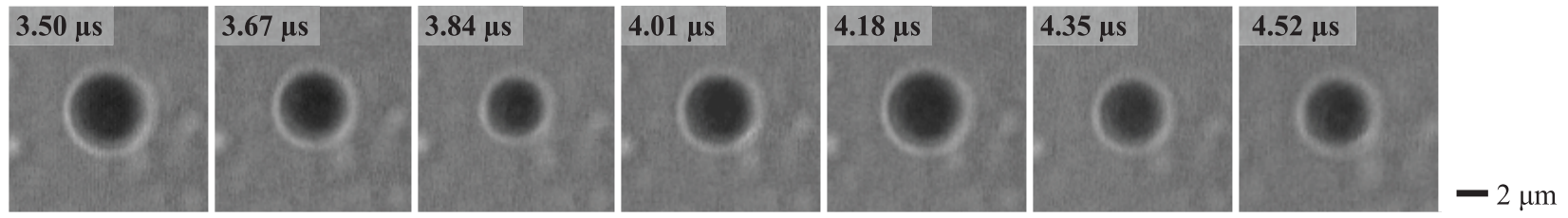

Fig. 5. Selected frames of a Brandaris 128 ultrahigh-speed camera recording of a microbubble oscillating in the OrganoPlate $\left(R_{0}=2.18 \mu \mathrm{m}\right)$ when insonified at $f_{T}=1.6 \mathrm{MHz}$ and $20-\mathrm{kPa}$ PNP.

a)

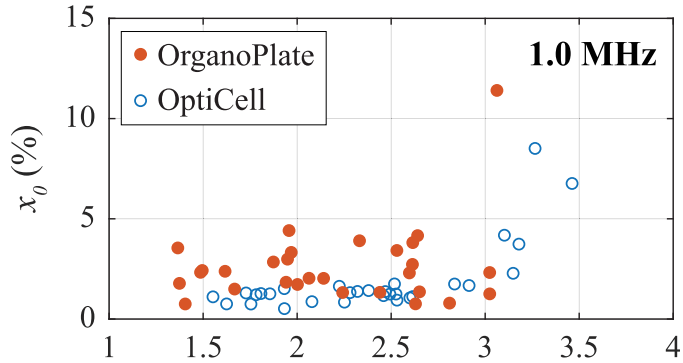

b)

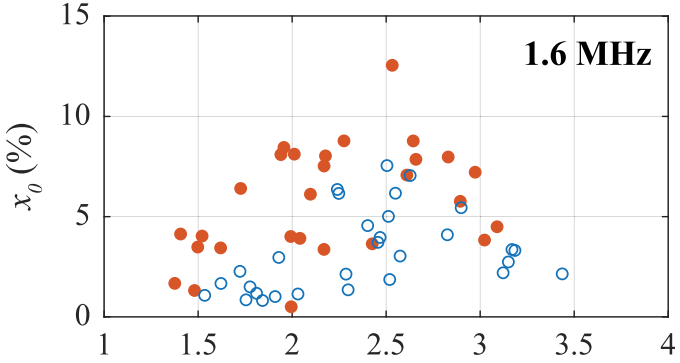

c)

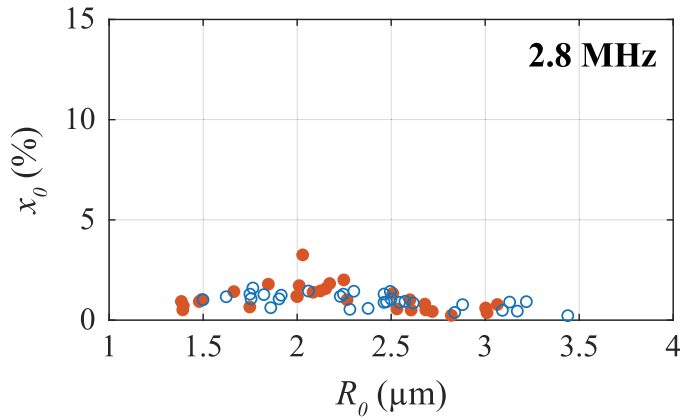

Fig. 6. Relative excursion amplitude $\left(x_{0}\right)$ of 29 single microbubbles in the OrganoPlate (solid red) and 30 single microbubbles in the OptiCell (open blue) as a function of resting radius $\left(R_{0}\right)$ when insonified at 20-kPa PNP for (a) $f_{T}=1 \mathrm{MHz}$, (b) $f_{T}=1.6 \mathrm{MHz}$ and (c) $f_{T}=2.8 \mathrm{MHz}$.

$f_{T}=1.6 \mathrm{MHz}$ is shown in Fig. 7(a). The iterative method clearly stabilizes within 30 iterations for both the OrganoPlate and the OptiCell. The frequency dependence of $P^{*}$ is shown in Fig. 7(b). The pressure transmitted into the OrganoPlate was larger than in the OptiCell for $f_{T}$ from 1 to $2 \mathrm{MHz}$, with mean $P^{*}=4.7 \mathrm{~dB}$ in the OrganoPlate and $P^{*}=-5.0 \mathrm{~dB}$ in the OptiCell. The mean $P^{*}$ from 2 to $4 \mathrm{MHz}$ was similar in both systems: $-6.1 \mathrm{~dB}$ in the OrganoPlate and $-5.1 \mathrm{~dB}$ in the OptiCell. In contrast to the OptiCell, the OrganoPlate showed a clear frequency-dependent behavior. The $\chi$ (with IQR between brackets), obtained by fitting $f_{0}$ (Fig. 8) to equation (2), was similar in both the OrganoPlate and the OptiCell, $\chi=0.36(0.35)$ and $0.32(0.25) \mathrm{N} / \mathrm{m}$, respectively. The median $\kappa_{s}$ (IQR) was also similar, $\kappa_{s}=1.1(0.6) \times$ $10^{-8} \mathrm{~kg} / \mathrm{s}$ in the OrganoPlate and $\kappa_{s}=0.7(0.4) \times 10^{-8} \mathrm{~kg} / \mathrm{s}$ in the OptiCell.

\section{B. Finite-Element Model}

The acoustic pressure as a function of time was simulated in water, in the OrganoPlate, and in the OptiCell (see examples depicted in Fig. 9). At all $f_{T}$, the acoustic pressure in water was evaluated as a reference for the free field and showed a clear start and endpoint of the pressure wave. In addition, the peak amplitude was constant at 20-kPa PNP over all eight cycles, with the exception of transient behavior in the first and last pulse.

At $f_{T}=1 \mathrm{MHz}$ [Fig. 9(a)], the pressure amplitude in the OrganoPlate was larger than that in the free field, with $P_{\text {sim }}=$ $26 \mathrm{kPa}$, homogeneous over all cycles, and $15 \%$ persisted after the intended eight cycles due to reverberations. At $f_{T}=$ 1.6 MHz [Fig. 9(b)], the pressure wave in the OrganoPlate was similar to that in water; the pressure amplitude was $P_{\text {sim }}=22 \mathrm{kPa}$, homogeneous over all cycles, and only $7 \%$ of $P_{\text {sim }}$ was observed after the intended pulse. At $f_{T}=2.8 \mathrm{MHz}$ [Fig. 9(c)], the pressure amplitude in the OrganoPlate was only $P_{\text {sim }}=14 \mathrm{kPa}$, but maintained its homogeneity among cycles and $6 \%$ of $P_{\text {sim }}$ remained. On the other hand, in the OptiCell the pressure amplitude was lower than that in the free field for all $f_{T}$. While the pressure was maintained homogeneous over all cycles, the amount of $P_{\text {sim }}$ persisting after the intended eight-cycle pulse increased from $8 \%$ at $f_{T}=1 \mathrm{MHz}$ up to $30 \%$ at $2.8 \mathrm{MHz}$.

Fig. 10 shows examples of the distribution of $P_{\text {sim }}^{*}$ in the free field, the OrganoPlate, and the OptiCell at $f_{T}=1 \mathrm{MHz}$ [Fig. 10(b), (e), and (h)], $f_{T}=$ $1.6 \mathrm{MHz}$ [Fig. 10(c), (f), and (i)], and $f_{T}=2.8 \mathrm{MHz}$ [Fig. 10(d), (g), and (j)]. The pressure wave was incident under $45^{\circ}$ from the positive $x$-axis, as schematically illustrated in Fig. 10(a). The pressure field in water had a clear elliptical focus with a smaller $-6 \mathrm{~dB}$ area for higher transmit frequencies [Fig. 10(e)-(g)]. Inside the OrganoPlate the elliptical focus was clearly changed; the redistribution of the pressure field shifted the maximum toward the right-hand side of the intended focus (positive $y$-axis). The normalized pressure amplitude when transmitting at $1 \mathrm{MHz}$ was $5.3 \mathrm{~dB}$ at the maximum and $2.3 \mathrm{~dB}$ in the focus [Fig. 10(b)], while $2.9 \mathrm{~dB}$ at the maximum and $0.7 \mathrm{~dB}$ in the focus for $1.6 \mathrm{MHz}$ [Fig. 10(c)]. At $2.8 \mathrm{MHz}$, it decreased to $-0.4 \mathrm{~dB}$ at the maximum and $-3.2 \mathrm{~dB}$ in the focus [Fig. 10(d)]. Finally, the pressure distribution in the OptiCell was only slightly more elongated in the direction of propagation than in the free field and the pressure amplitude was at least $-2.7 \mathrm{~dB}$ lower than $P_{\text {ref }}$ [Fig. 10(h)-(j)].

The frequency dependence of the simulated pressure normalized to the free field is shown in Fig. 11, for both the 


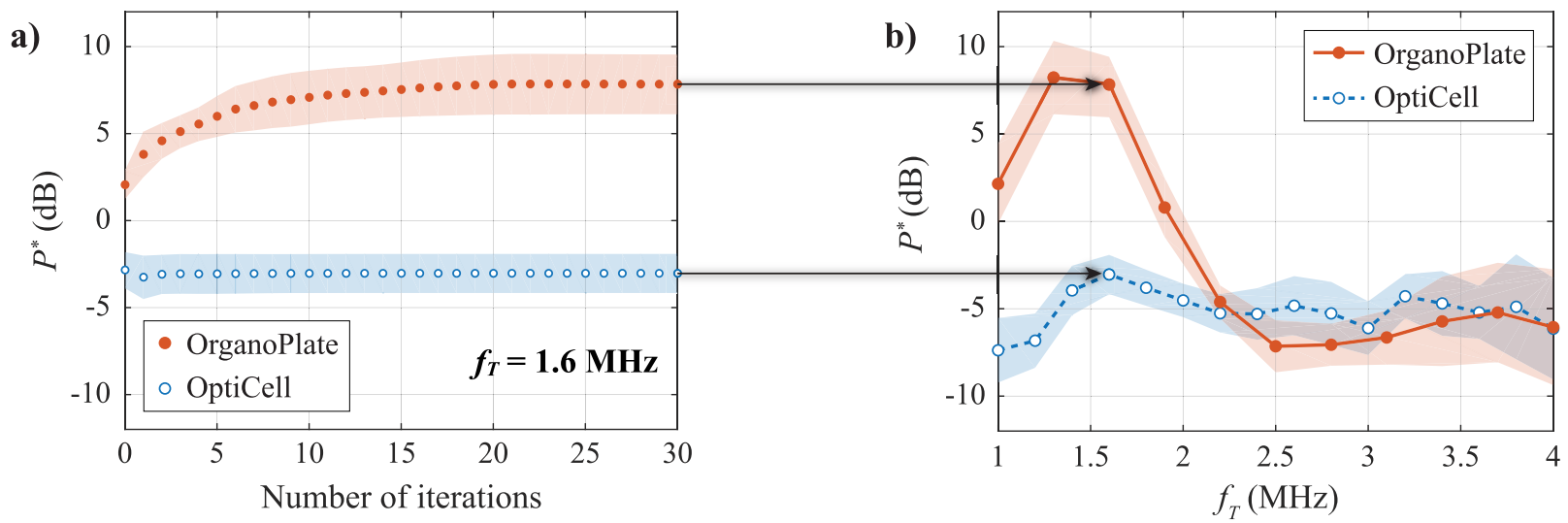

Fig. 7. Normalized pressure $\left(P^{*}\right)$ experimentally determined in the OrganoPlate (solid red) and OptiCell (open/dashed blue). The shaded area corresponds to the IQR. (a) Progress and stabilization of $P^{*}$ with increasing number of iterations, when insonified at $f_{T}=1.6 \mathrm{MHz}$. (b) Final $P^{*}$ obtained at iteration 30 , as a function of $f_{T}$. The arrows indicate the final $P^{*}$ as plotted in (a).

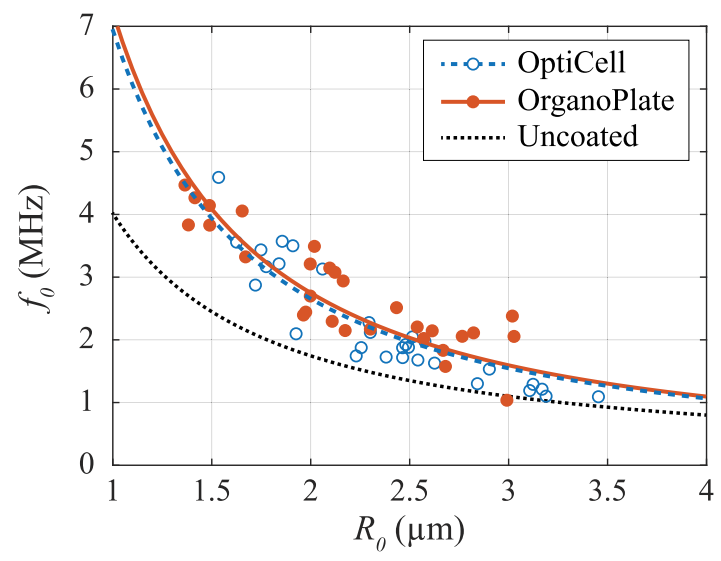

Fig. 8. Eigenfrequency $\left(f_{0}\right)$ of microbubbles in the OrganoPlate (solid red circles) and OptiCell (open blue circles) and the corresponding fit to (2), in order to obtain the average shell elasticity. The $f_{0}$ of an uncoated microbubble is given by $\chi=0 \mathrm{~N} / \mathrm{m}$ (black dotted line).

OrganoPlate and the OptiCell. For $f_{T}$ between 1 and $2 \mathrm{MHz}$, the mean $P_{\text {sim }}^{*}$ in the OrganoPlate was higher than in the OptiCell (1.6 and $-2.8 \mathrm{~dB}$, respectively). For $f_{T}$ from 2 to $4 \mathrm{MHz}$, the mean $P_{\text {sim }}^{*}$ in both systems was similar, with $-4 \mathrm{~dB}$ in the OrganoPlate and $-3.8 \mathrm{~dB}$ in the OptiCell. For comparison, the $P^{*}$ obtained experimentally [Fig. 7(b)] is also shown in Fig. 11.

\section{DISCUSSION}

To the best of our knowledge, the OrganoPlate has never been used before in combination with ultrasound. Moreover, no information was available on acoustic propagation into the microchannels, which is essential to understand and predict microbubble behavior. The small dimensions of the microchannels in the OrganoPlate do not allow direct measurements of the pressure field using a hydrophone without disturbing the ultrasound field. We therefore assessed the feasibility of controlled microbubble behavior in the OrganoPlate by experimentally studying microbubble oscillation and modeling the pressure wave propagation. We found that the pressure wave successfully propagated into the microchannels of the a)

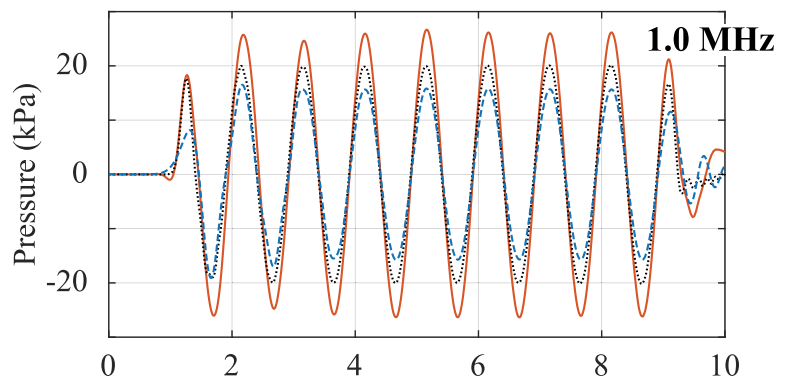

b)

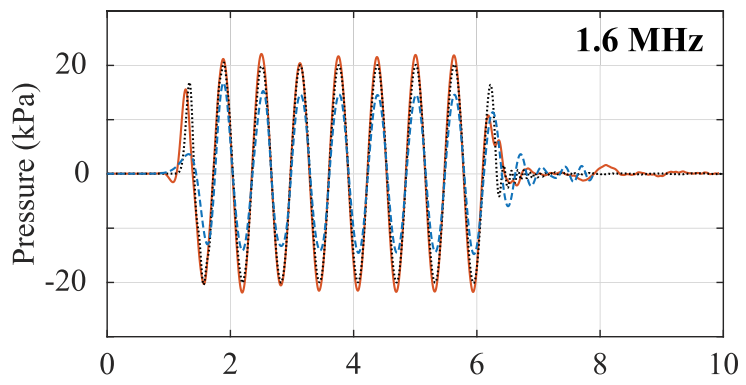

c)

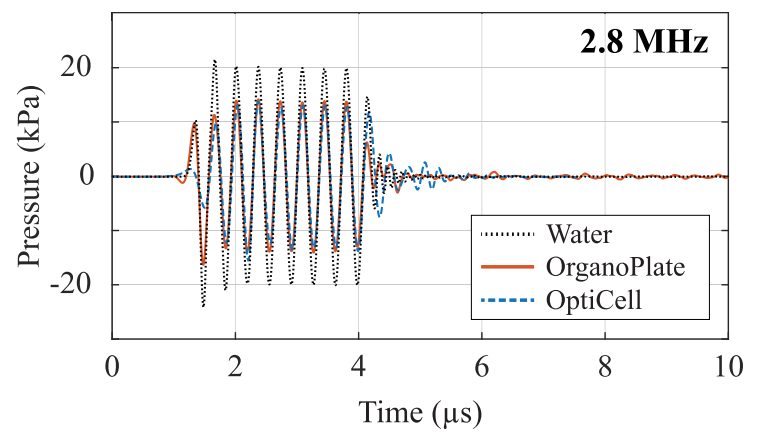

Fig. 9. Acoustic pressure as a function of time at the focus in water (dotted black line), the OrganoPlate (solid red line), and the OptiCell (dashed blue line) simulated with the 3-D finite-element model transmitting eight cycles at (a) $f_{T}=1 \mathrm{MHz}$, (b) $f_{T}=1.6 \mathrm{MHz}$, or (c) $f_{T}=2.8 \mathrm{MHz}$.

OrganoPlate and resulted in a predictable microbubble oscillatory response.

Microbubble behavior upon ultrasound insonification was fit to the model of a harmonic oscillator. Measurements were performed at $20-\mathrm{kPa}$ PNP in order to keep the intrinsic nonlinear microbubble response to a minimum [32]. However, 

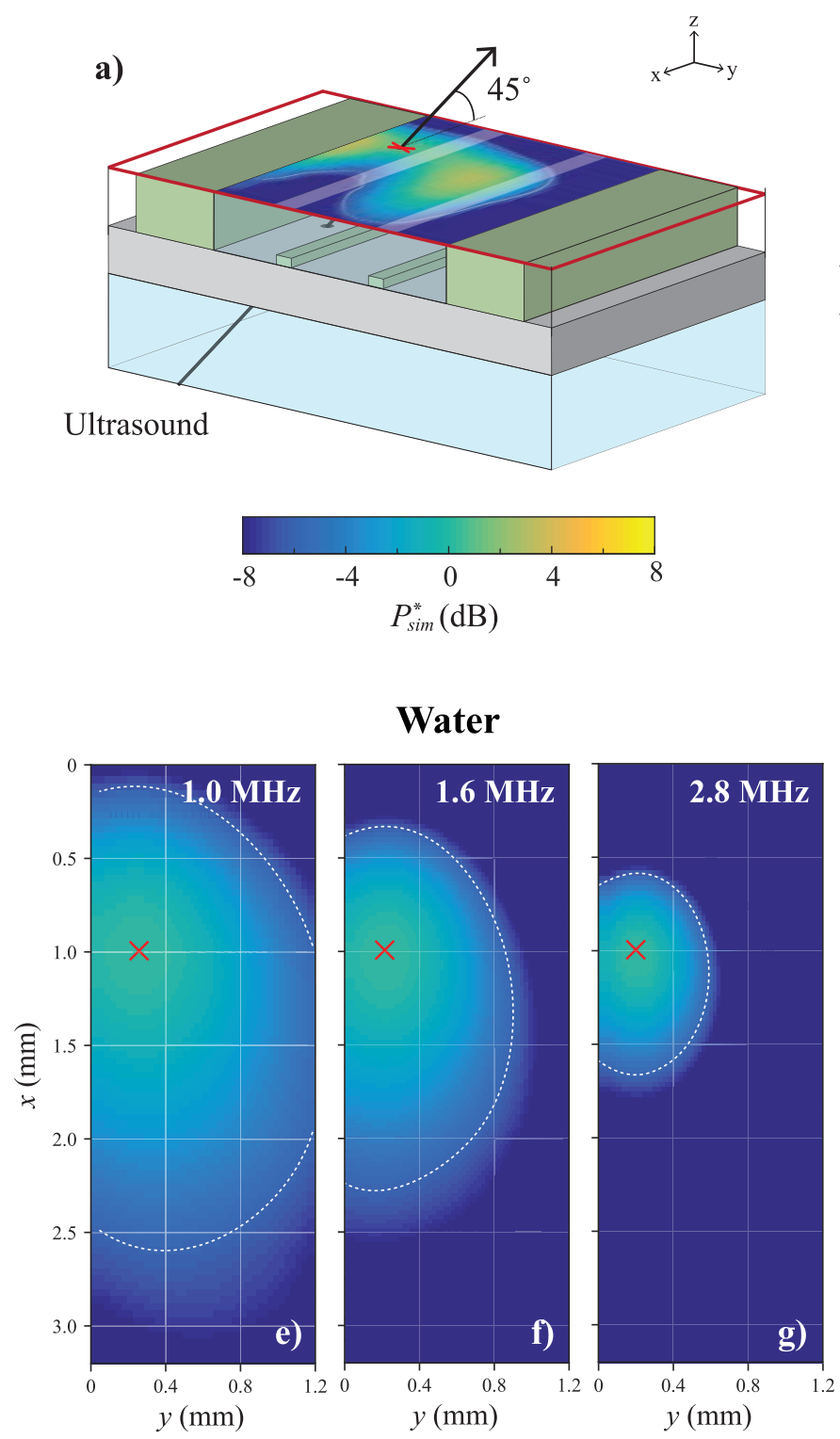

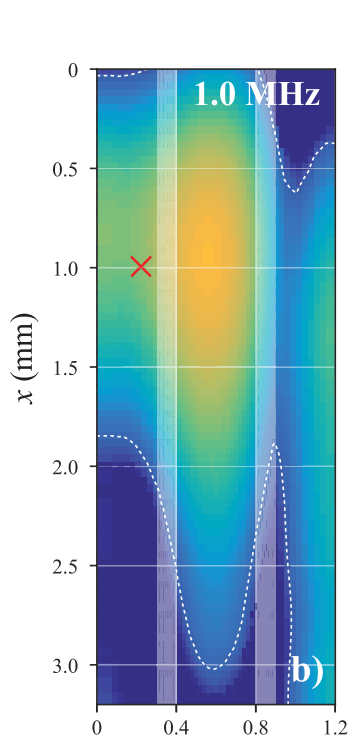

\section{OrganoPlate}

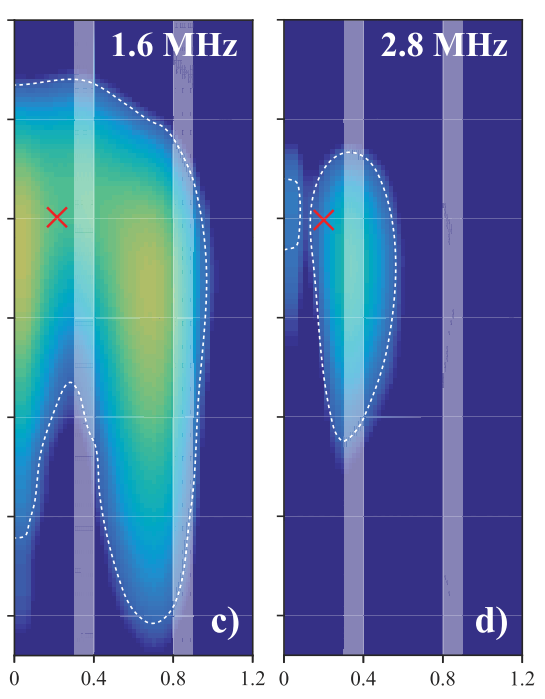

OptiCell
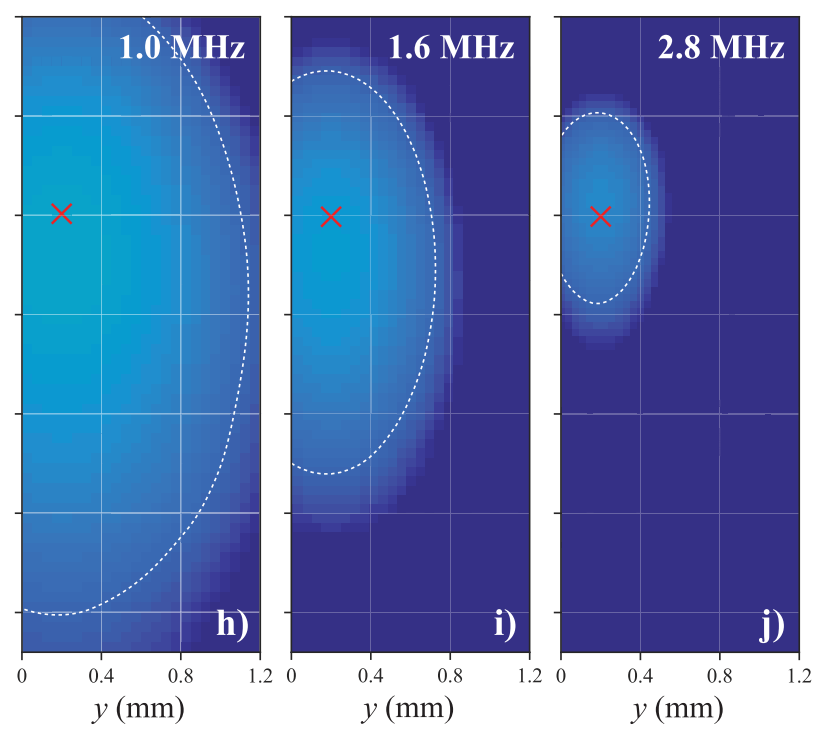

Fig. 10. Normalized pressure $\left(P_{\text {sim }}^{*}\right)$ in the $x y$-plane at the axial focus simulated with the 3-D finite-element model, obtained by normalizing the pressure amplitude $\left(P_{\text {sim }}\right)$ to the maximum pressure amplitude when transmitted in water $\left(P_{\text {ref }}\right)$. The -6 dB contours are indicated by the white dashed line, the ultrasound geometrical focus by the red cross, and the phaseguides of the OrganoPlate by the white bands. (a) Schematic of the OrganoPlate illustrating the $x y$-plane (delineated in red) at which $P_{\text {sim }}^{*}$ was determined and the ultrasound incidence direction. This example shows $P_{\text {sim }}^{*}$ at $f_{T}=1.6 \mathrm{MHz}$. The $P_{\text {sim }}^{*}$ in the OrganoPlate is shown at (b) $f_{T}=1$, (c) 1.6 , and (d) $2.8 \mathrm{MHz}$; in water at (e) $f_{T}=1$, (f) 1.6 , and (g) $2.8 \mathrm{MHz}$; and in the OptiCell at (h) $f_{T}=1$, (i) 1.6 , and (j) $2.8 \mathrm{MHz}$.

the viscoelastic shell parameters are pressure-dependent [28]. With our iterative method, we accounted for this nonlinear viscoelastic behavior as the shell was characterized for each individual microbubble. Hence, allowing for a possible change in shell elasticity and viscosity with pressure. Nevertheless, our method does not account for variation of shell parameters during microbubble oscillation itself. Instead, effective shell parameters are obtained that approximate these variations without characterizing the different contributions. The goal of our iterative method is to determine the pressure experienced by the microbubbles, and the effective shell parameters allow us to predict their behavior using the current physical models.
The spread of the $x_{0}$ data points (Fig. 6) indicates variations among microbubbles: two microbubbles of the same size may have very different relative excursions. Variations amongst microbubbles of identical size have previously been observed for microbubbles with equal and different coating compositions [18], [32], [41], [42]. While the mean microbubble shell properties are often used to describe microbubble dynamics [7], [28], [31], [32], we determined $f_{0}$ and $\delta$ specifically for each individual microbubble. By incorporating the variation of shell properties among microbubbles, we were able to more accurately describe the behavior of each individual microbubble. 


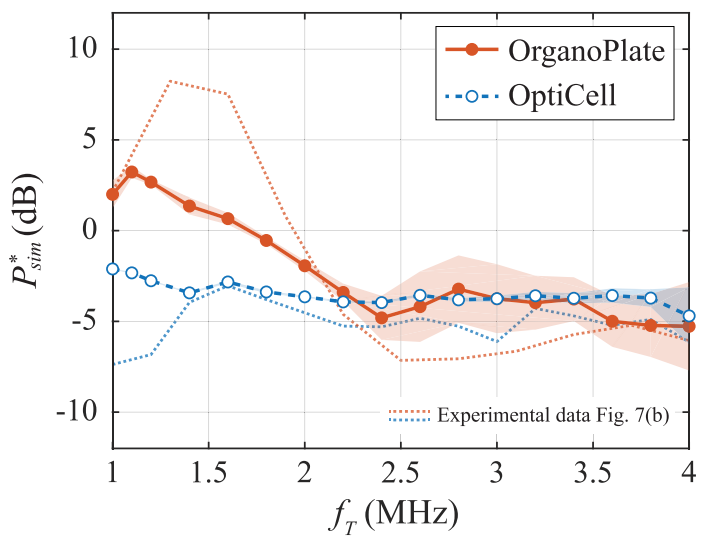

Fig. 11. Normalized pressure $\left(P_{\text {sim }}^{*}\right)$ at the focus in the OrganoPlate (red solid line) and OptiCell (blue dashed line) as a function of $f_{T}$, simulated with the 3-D finite-element models. The shaded area represents the IQR. The dotted lines show the mean $P^{*}$ obtained experimentally, as shown in Fig. 7(b).

In this paper, the ultrasound propagation into the OrganoPlate was compared to that of the OptiCell, since the latter is commonly used in ultrasound-mediated drug delivery studies. Although the OptiCell has been extensively used in microbubble characterization and ultrasound-mediated drug delivery studies, a thorough acoustic characterization has not been published. Often, the OptiCell is assumed to be acoustically transparent [4], [28], [43], [44] despite the attenuation that was observed in our experiments and simulations. The main difference between the OrganoPlate and the OptiCell was found for incidence frequencies between 1 and $2 \mathrm{MHz}$, where the pressure amplitude in the OrganoPlate was higher. Nevertheless, when comparing the OrganoPlate to the OptiCell from 2 to $4 \mathrm{MHz}$, the simulations predict a root-mean-square deviation (RMSD) of only $0.9 \mathrm{~dB}$ and the measurements a RMSD of $1.4 \mathrm{~dB}$ between both systems. Considering that our experimental data was influenced by at least a $10 \%$ error of the tracking algorithm [31] and a 15\% uncertainty of the hydrophone for the transducer calibration, the pressure in the OrganoPlate and OptiCell between 2 and $4 \mathrm{MHz}$ was not significantly different. Furthermore, the characteristic frequency response of microbubbles was unchanged since $f_{0}$ and $\kappa_{s}$ were the same in both the OrganoPlate and the OptiCell.

The focal region in the OrganoPlate was clearly altered (Fig. 10) because of the reflections at the polymer walls and air spacing outside of the microchannels. The parallel membranes of the OptiCell, on the other hand, had a minor effect on the shape of the focus. Nevertheless, as long as this altered focal region is taken into account during the design of future experiments, microbubbles in the OrganoPlate can still be insonified in a controlled manner. To achieve reproducible insonifications, standing waves should be avoided [45]. In other words, we want a quick buildup and decay of the intended eight-cycle pulse. By modeling the pressure field in the OrganoPlate, we found no significant remaining oscillations after the intended pulse. On the other hand, the pressure amplitude observed in the OptiCell after the eight-cycle pulse was slightly larger than in the OrganoPlate. Hence, energy was transported better into and out of the OrganoPlate and no standing wave phenomenon was observed.
When comparing the microbubble spectroscopy measurements to the finite-element models, the transmitted pressure from 1 to $2 \mathrm{MHz}$ showed the largest deviation. In this frequency range, the RMSD between measurements and simulations was $4.4 \mathrm{~dB}$ for the OrganoPlate and $3 \mathrm{~dB}$ for the OptiCell. Although both approaches clearly showed higher pressures in the OrganoPlate, the experimental data analysis overestimated this effect. A possible explanation is that a single shell elasticity value was determined for every microbubble based on the full range of transmit frequencies. However, at low frequencies the pressure experienced by the microbubble in the OrganoPlate is larger and $\chi$ is overestimated, since the effective shell elasticity should decrease with increasing pressure [28]. An overestimation in shell elasticity implies a stiffer shell and would thus lead to smaller relative excursion amplitudes. Therefore, when fitting our measurements to the model of a linear oscillator, the pressure amplitude is overestimated at low transmit frequencies. Nevertheless, when transmitting between 2 and $4 \mathrm{MHz}$, the RMSD between the simulations and the measurements is only $2 \mathrm{~dB}$ for the OrganoPlate and $1.4 \mathrm{~dB}$ for the OptiCell.

In both the measurements and simulations, the pressure transmitted into the OrganoPlate showed a clear frequencydependent behavior. For insonifications between 1 and $2 \mathrm{MHz}$, the mean of the measured and simulated pressure amplitude was about $3 \mathrm{~dB}$ larger than in the free field. Between 2 and $4 \mathrm{MHz}$, the pressure in the OrganoPlate decreased to $-5 \mathrm{~dB}$. Frequency-dependent behavior is expected, since different wavelengths result in different wave interference patterns caused by the dimensions of the OrganoPlate. In the future experiments, ultrasound insonification can be corrected for these changes in pressure as a function of frequency. For instance, to obtain $100 \mathrm{kPa}$ inside the OrganoPlate at $1 \mathrm{MHz}$, $71 \mathrm{kPa}$ needs to be transmitted, while this is $178 \mathrm{kPa}$ at $3 \mathrm{MHz}$. On the other hand, the pressure propagated into the OptiCell showed no clear frequency dependence from 1 to $4 \mathrm{MHz}$, with a mean normalized pressure of measurements and simulations of $-4 \mathrm{~dB}$.

A limitation of this paper is that only the propagation of an eight-cycle pulse was considered. Although some studies employ short pulses to study ultrasound-mediated drug delivery [12], [18], [46], others use much longer bursts to locally induce drug uptake [4], [13], [15], [47]. However, the finite-element model showed minimal oscillations persisting after the eight cycles, suggesting that energy was easily transported out of the OrganoPlate and therefore longer pulses are not expected to affect the pressure amplitude. Another shortcoming of this paper is that the pressure field was only studied in the $x y$-plane just beneath the top glass plate. When targeting microbubbles to a microvessel grown in the OrganoPlate, insonification in a different $x y$-plane may be required. Since the beamwidth of the incident ultrasound field is large with respect to the small microchannels, the exact focal location in the OrganoPlate is expected to only be of minor influence on the pressure field. If required, the now validated 3-D finite-element model can be employed to predict the pressure field at any location within the OrganoPlate. Finally, when using the modified OrganoPlate to study ultrasound-mediated 
drug delivery, biological aspects such as cell culture protocols may need to be reconsidered.

\section{CONCLUSION}

The feasibility of controlled microbubble oscillation in the OrganoPlate was demonstrated by microbubble spectroscopy and finite-element modeling of the acoustic pressure propagation. When transmitting from 1 to $2 \mathrm{MHz}$, the pressure amplitude inside the OrganoPlate was about $3 \mathrm{~dB}$ larger than in the free field. On the other hand, when transmitting between 2 and $4 \mathrm{MHz}$ the pressure amplitude was approximately $-5 \mathrm{~dB}$ with respect to that of the free field, similar to $-4 \mathrm{~dB}$ in the OptiCell. When correcting for the known change in pressure, controlled microbubble behavior can be achieved in the OrganoPlate. This demonstrates the potential of the OrganoPlate to study ultrasound-mediated drug delivery in vitro including 3-D cell culture, perfusion, and membranefree soft boundaries.

\section{ACKNOWLEDGMENT}

The authors would like to thank F. Mastik and R. Beurskens from the Department of Biomedical Engineering, Erasmus MC, Rotterdam, The Netherlands, for their technical assistance during the experiments.

\section{REFERENCES}

[1] K. Kooiman, H. J. Vos, M. Versluis, and N. de Jong, "Acoustic behavior of microbubbles and implications for drug delivery," Adv. Drug Delivery Rev., vol. 72, pp. 28-48, Jun. 2014.

[2] I. Lentacker, I. De Cock, R. Deckers, S. C. De Smedt, and C. T. W. Moonen, "Understanding ultrasound induced sonoporation: Definitions and underlying mechanisms," Adv. Drug Delivery Rev. vol. 72, pp. 49-64, Jun. 2014.

[3] A. van Wamel et al., "Vibrating microbubbles poking individual cells: Drug transfer into cells via sonoporation," J. Controlled Release, vol. 112, no. 2, pp. 149-155, May 2006.

[4] B. D. M. Meijering et al., "Ultrasound and microbubble-targeted delivery of macromolecules is regulated by induction of endocytosis and pore formation," Circulation Res., vol. 104, no. 5, pp. 679-687, Mar. 2009.

[5] N. Sheikov, N. McDannold, S. Sharma, and K. Hynynen, "Effect of focused ultrasound applied with an ultrasound contrast agent on the tight junctional integrity of the brain microvascular endothelium," Ultrasound Med. Biol., vol. 34, no. 7, pp. 1093-1104, 2008.

[6] H. J. Vos, B. Dollet, M. Versluis, and N. de Jong, "Nonspherical shape oscillations of coated microbubbles in contact with a wall," Ultrasound Med. Biol., vol. 37, no. 6, pp. 935-948, Jun. 2011.

[7] M. Overvelde, V. Garbin, B. Dollet, N. de Jong, D. Lohse, and M. Versluis, "Dynamics of coated microbubbles adherent to a wall," Ultrasound Med. Biol., vol. 37, no. 9, pp. 1500-1508, Sep. 2011.

[8] B. L. Helfield, B. Y. C. Leung, and D. E. Goertz, "The effect of boundary proximity on the response of individual ultrasound contrast agent microbubbles," Phys. Med. Biol., vol. 59, no. 7, pp. 1721-1745, 2014.

[9] Y. Liu, E. Gill, and Y. Y. S. Huang, "Microfluidic on-chip biomimicry for 3D cell culture: A fit-for-purpose investigation from the end user standpoint," Future Sci., vol. 3, no. 2, p. FSO173, 2017.

[10] F. Pampaloni, E. G. Reynaud, and E. H. K. Stelzer, "The third dimension bridges the gap between cell culture and live tissue," Nature Rev. Mol. Cell Biol., vol. 8, no. 10, pp. 839-845, 2007.

[11] T. G. Walsh et al., "Stabilization of brain microvascular endothelial barrier function by shear stress involves VE-cadherin signaling leading to modulation of pTyr-occludin levels," J. Cellular Physiol., vol. 226, no. 11, pp. 3053-3063, Nov. 2011.

[12] K. Kooiman, M. Foppen-Harteveld, A. F. W. van der Steen, and N. de Jong, "Sonoporation of endothelial cells by vibrating targeted microbubbles," J. Controlled Release, vol. 154, no. 1, pp. 35-41, Aug. 2011.
[13] I. De Cock et al., "Ultrasound and microbubble mediated drug delivery: Acoustic pressure as determinant for uptake via membrane pores or endocytosis," J. Controlled Release, vol. 197, pp. 20-28, Jan. 2015.

[14] A. Yudina et al., "Ultrasound-mediated intracellular drug delivery using microbubbles and temperature-sensitive liposomes," J. Controlled Release, vol. 155, no. 3, pp. 442-448, Nov. 2011.

[15] T. van Rooij et al., "Viability of endothelial cells after ultrasoundmediated sonoporation: Influence of targeting, oscillation, and displacement of microbubbles," J. Controlled Release, vol. 238, pp. 197-211, Sep. 2016

[16] I. Lentacker, B. Geers, J. Demeester, S. C. De Smedt, and N. N. Sanders, "Design and evaluation of doxorubicin-containing microbubbles for ultrasound-triggered doxorubicin delivery: Cytotoxicity and mechanisms involved," Molecular Therapy, vol. 18, no. 1, pp. 101-108, 2010.

[17] Z. Fan, R. E. Kumon, J. Park, and C. X. Deng, "Intracellular delivery and calcium transients generated in sonoporation facilitated by microbubbles," J. Controlled Release, vol. 142, no. 1, pp. 31-39, 2010.

[18] B. Helfield, X. Chen, S. C. Watkins, and F. S. Villanueva, "Biophysical insight into mechanisms of sonoporation," Proc. Nat. Acad. Sci. USA vol. 113, no. 36, pp. 9983-9988, 2016.

[19] J. Park, Z. Fan, and C. X. Deng, "Effects of shear stress cultivation on cell membrane disruption and intracellular calcium concentration in sonoporation of endothelial cells," J. Biomech., vol. 44, no. 1, pp. 164-169, Jan. 2011.

[20] F. E. Shamout et al., "Enhancement of non-invasive trans-membrane drug delivery using ultrasound and microbubbles during physiologically relevant flow," Ultrasound Med. Biol., vol. 41, no. 9, pp. 2435-2448, Sep. 2015.

[21] D. Carugo, J. Owen, C. Crake, J. Y. Lee, and E. Stride, "Biologically and acoustically compatible chamber for studying ultrasound-mediated delivery of therapeutic compounds," Ultrasound Med. Biol., vol. 41, no. 7, pp. 1927-1937, Jul. 2015.

[22] V. van Duinen, S. J. Trietsch, J. Joore, P. Vulto, and T. Hankemeier, "Microfluidic 3D cell culture: From tools to tissue models," Current Opinion Biotechnol., vol. 35, pp. 118-126, Dec. 2015.

[23] S. J. Trietsch, G. D. Israëls, J. Joore, T. Hankemeier, and P. Vulto, "Microfluidic titer plate for stratified 3D cell culture," Lab Chip, vol. 13 no. 18 , pp. 3548-3554, 2013

[24] P. Vulto, S. Podszun, P. Meyer, C. Hermann, A. Manz, and G. A. Urban, "Phaseguides: A paradigm shift in microfluidic priming and emptying," Lab Chip, vol. 11, no. 9, pp. 1596-1602, 2011.

[25] N. R. Wevers et al., "High-throughput compound evaluation on 3D networks of neurons and glia in a microfluidic platform," Sci. Rep., vol. 6, Dec. 2016, Art. no. 38856.

[26] S. J. Trietsch et al., "Membrane-free culture and real-time barrier integrity assessment of perfused intestinal epithelium tubes," Nature Commun., vol. 8, no. 1, Aug. 2017, Art. no. 262.

[27] M. Jang, P. Neuzil, T. Volk, A. Manz, and A. Kleber, "On-chip threedimensional cell culture in phaseguides improves hepatocyte functions in vitro," Biomicrofluidics, vol. 9, no. 3, p. 34113, May 2015.

[28] M. Overvelde et al., "Nonlinear shell behavior of phospholipid-coated microbubbles," Ultrasound Med. Biol., vol. 36, no. 12, pp. 2080-2092, Dec. 2010.

[29] T. Segers, L. de Rond, N. de Jong, M. Borden, and M. Versluis, "Stability of monodisperse phospholipid-coated microbubbles formed by flow-focusing at high production rates," Langmuir, vol. 32, no. 16, pp. 3937-3944, 2016

[30] C. T. Chin et al., "Brandaris 128: A digital 25 million frames per second camera with 128 highly sensitive frames," Rev. Sci. Instrum., vol. 74, no. 12, pp. 5026-5034, 2003.

[31] S. M. van der Meer et al., "Microbubble spectroscopy of ultrasound contrast agents," J. Acoust. Soc. Amer., vol. 121, no. 1, pp. 648-656, 2007.

[32] T. van Rooij et al., "Non-linear response and viscoelastic properties of lipid-coated microbubbles: DSPC versus DPPC," Ultrasound Med. Biol., vol. 41, no. 5, pp. 1432-1445, May 2015

[33] K. Kooiman et al., "DSPC or DPPC as main shell component influences ligand distribution and binding area of lipid-coated targeted microbubbles," Eur. J. Lipid Sci. Technol., vol. 116, no. 9, pp. 1217-1227, Sep. 2014

[34] A. L. Klibanov et al., "Detection of individual microbubbles of ultrasound contrast agents: Imaging of free-floating and targeted bubbles," Invest. Radiol., vol. 39, no. 3, pp. 187-195, 2004.

[35] T. G. Leighton, The Acoustic Bubble. London, U.K.: Academic, 1994 
[36] S. Hilgenfeldt, D. Lohse, and M. Zomack, "Response of bubbles to diagnostic ultrasound: A unifying theoretical approach," Eur. Phys. J. B, Condense. Matter Complex Syst., vol. 4, no. 2, pp. 247-255, Aug. 1998.

[37] Schott D263 Thin Borosilicate Glass, MatWeb. [Online]. Available: http://www.matweb.com/search/datasheettext.aspx?matguid= 8df9f3e0106d43818ebe1862e76a1107

[38] D. Vaughn and J. Mould, PZFlex Time Domain Finite Element Analysis Package. Cupertino, CA, USA: PZFlex LLC, 2001.

[39] W. Xia, D. Piras, J. C. G. van Hespen, W. Steenbergen, and S. Manohar, "A new acoustic lens material for large area detectors in photoacoustic breast tomography," Photoacoustics, vol. 1, no. 2, pp. 9-18, May 2013.

[40] G. S. Kino, Acoustic Waves: Devices, Imaging, and Analog Signal Processing, vol. 100. Englewood Cliffs, NJ, USA: Prentice-Hall, 1987.

[41] K. Kooiman et al., "Focal areas of increased lipid concentration on the coating of microbubbles during short tone-burst ultrasound insonification," PLoS ONE, vol. 12, no. 7, p. e0180747, Jul. 2017.

[42] M. Emmer, H. J. Vos, M. Versluis, and N. de Jong, "Radial modulation of single microbubbles," IEEE Trans. Ultrason., Ferroelect., Freq. Control, vol. 56, no. 11, pp. 2370-2379, Nov. 2009.

[43] A. Rahim, S. L. Taylor, N. L. Bush, G. R. ter Haar, J. C. Bamber, and C. D. Porter, "Physical parameters affecting ultrasound/microbubblemediated gene delivery efficiency in vitro," Ultrasound Med. Biol., vol. 32, no. 8, pp. 1269-1279, Aug. 2006.

[44] J. Sijl et al., "Subharmonic behavior of phospholipid-coated ultrasound contrast agent microbubbles," J. Acoust. Soc. Amer., vol. 128, no. 5, pp. 3239-3252, 2010.

[45] K. Hensel, M. P. Mienkina, and G. Schmitz, "Analysis of ultrasound fields in cell culture wells for in vitro ultrasound therapy experiments," Ultrasound Med. Biol., vol. 37, no. 12, pp. 2105-2115, 2011.

[46] Y. Hu, J. M. F. Wan, and A. C. H. Yu, "Membrane perforation and recovery dynamics in microbubble-mediated sonoporation," Ultrasound Med. Biol., vol. 39, no. 12, pp. 2393-2405, Dec. 2013.

[47] K. Kooiman, M. Emmer, M. Foppen-Harteveld, A. van Wamel, and N. de Jong, "Increasing the endothelial layer permeability through ultrasound-activated microbubbles," IEEE Trans. Biomed. Eng., vol. 57, no. 1, pp. 29-32, Jan. 2010.

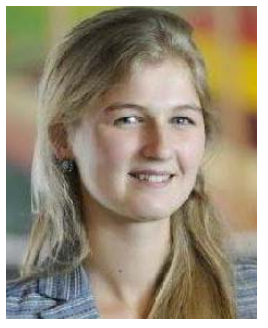

Inés Beekers (S'16) received the B.Sc. and M.Sc degrees in applied physics with a specialization in medical imaging and acoustical wavefield modeling from Delft University of Technology, Delft, The Netherlands, in 2013 and 2015, respectively. She is currently pursuing the Ph.D. degree with the Department of Biomedical Engineering, Erasmus Medical Center, Rotterdam, The Netherlands. Her thesis focuses on unraveling the underlying mechanisms involved in ultrasound-mediated vascular drug delivery with contrast agents, using both experimental and modeling approaches.

She was an Intern at the Organ-on-a-Chip company Mimetas B.V., Leiden, The Netherlands.

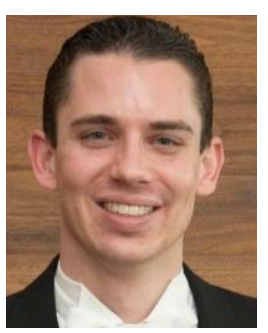

Tom van Rooij (S'15-M'16) was born in Eindhoven, The Netherlands, in 1987. He received the B.Sc. degree in biomedical engineering and the M.Sc. degree in medical engineering from the Eindhoven University of Technology, Eindhoven, in 2008 and 2012, respectively, and the Ph.D. degree from the Department of Biomedical Engineering, Thorax Center, Erasmus MC, Rotterdam, The Netherlands, in 2017. His thesis focused on contrast agents for ultrasound imaging and therapy by using ultrahigh-speed optical imaging techniques and in vitro and in vivo ultrasound imaging.

He held a teaching position at the Biomedical NMR Group, Department of Biomedical Engineering, Eindhoven University of Technology. He is currently working as Application Scientist with the MILabs B.V., Utrecht, The Netherlands.

Dr. van Rooij received the Thudichum Award for his thesis by the Phospholipid Research Center during the 5th International Symposium on Phospholipids in Pharmaceutical Research, Heidelberg, Germany.

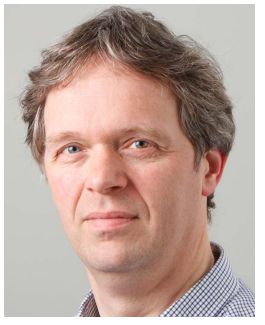

Martin D. Verweij (M'10) received the M.Sc. (cum laude) and Ph.D. degrees in electrical engineering from Delft University of Technology, Delft, The Netherlands, in 1988 and 1992, respectively.

From 1993 to 1997, he was a Research Fellow with the Prestigious Royal Netherlands Academy of Arts and Sciences, Amsterdam, The Netherlands. In 1995 and 1997, he was a Visiting Scientist at Schlumberger Cambridge Research, Cambridge, U.K. In 1998, he joined the Laboratory of Electromagnetic Research, Delft University of Technology, as an Associate Professor and later became an Assistant Professor, where he was with the Laboratory of Acoustical Wavefield Imaging in 2011. Since 2015, he has been holding a part-time position at the Biomedical Engineering Group, Erasmus Medical Centre, Rotterdam, The Netherlands. He is the originator of the Iterative Nonlinear Contrast Source (INCS) method for the computation of nonlinear ultrasound fields. He is a Research Leader with the Dutch Technology Foundation (STW), Utrecht, The Netherlands, where he is focusing on projects involving transducer design, beamforming, and imaging. His research interests include dedicated transducer design, beamforming algorithms, and the theoretical modeling and numerical simulation of medical ultrasound.

Dr. Verweij is an Associate Editor of the Journal of the Acoustical Society of America, and the Treasurer of the Dutch Society for Medical Ultrasound. He was elected the "Best Teacher of the Electrical Engineering curriculum" in 2001-2002.

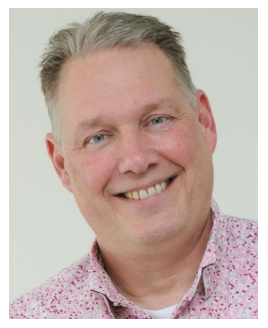

Michel Versluis (M'12) was born in The Netherlands in 1963. He received the M.Sc. degree in physics from the University of Nijmegen, Nijmegen, The Netherlands, with a special interest in molecular physics and astrophysics, involved in the field of farinfrared laser spectroscopy of interstellar molecular species. Later, he specialized in the application of intense tunable ultraviolet lasers for flame diagnostics, resulting in a successful defense of his Ph.D. thesis in 1992.

After a two-year research position working on molecular dynamics at Griffith University, Brisbane, QLD, Australia, he continued to focus on developing laser diagnostic techniques for internal combustion engines (Lund, Sweden) and industrial jet flames and solid rocket propellants (Delft, The Netherlands). He is currently a Full Professor of physical and medical acoustics with the Physics of Fluids Group, University of Twente, Enschede, The Netherlands. He is an expert in ultrahigh-speed imaging with a particular interest in the use of microbubbles and microdroplets for medical applications, both in imaging and in therapy, and in the physics and control of bubbles and droplets in microfluidic applications in medicine and the nanotechnology industry.

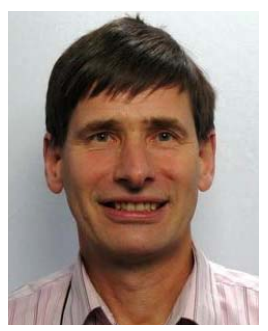

Nico de Jong (A'97-M'09) received the M.Sc. degree in physics specialized in the field of pattern recognition from Delft University of Technology, Delft, The Netherlands, in 1978, and the Ph.D degree with a specialization in acoustic properties of ultrasound contrast agents from the Department of Biomedical Engineering, Thorax Center, Erasmus Medical Center (Erasmus MC), Rotterdam, The Netherlands, in 1993

In 2003, he joined the University of Twente, Enschede, The Netherlands, as a part-time Professor. He currently teaches at Technical Universities and Erasmus MC. He has been a Promotor of $21 \mathrm{Ph} . \mathrm{D}$. students and is currently co-supervising $11 \mathrm{Ph} . \mathrm{D}$. students. Since 1980, he has been a Staff Member of the Thorax Center, Erasmus MC. Since 2011, he has been a Professor of Molecular Ultrasonic Imaging and Therapy with Erasmus MC and Delft University of Technology. $\mathrm{He}$ has been acquiring over $6 \mathrm{Me}$ as a Principal Investigator (PI) or a Co-PI since 2010. Since 2015, he has been the part-time Head of the Department of Acoustical Waveform Imaging with Delft University of Technology. Over the last five years, he has given more than 30 invited lectures and numerous scientific presentations for international industries. His H-factor is 49 (Web of science). He has authored 260 peer-reviewed articles and has been a PI and the Workpackage Leader of the European and Dutch projects.

Dr. de Jong is a member of the Safety Committee of the World Federation of Ultrasound in Medicine and Biology and the Organizer of the Annual European Symposium on Ultrasound Contrast Imaging, Rotterdam, which is attended by approximately 175 scientists from universities and industries all over the world. He is an Associate Editor of Ultrasound in Medicine and Biology, and a Guest Editor of the special issues of different journals. 


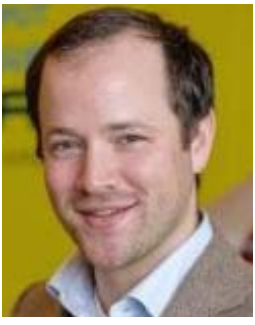

Sebastiaan J. Trietsch was born in The Hague, The Netherlands, in 1985. He received the M.Sc. degree in biopharmaceutical sciences, with a specialization in analytical chemistry, from the Leiden Academic Center for Drug Research (LACDR), Leiden University, Leiden, The Netherlands, and the Ph.D. degree from the Division of Analytical Biosciences, LACDR, with a focus on microfluidic technology for 3-D cell culture and resulted in the development of the OrganoPlate, a microfluidic platform for high throughput 3-D cell culture.

He was with the Faculty of Pharmacy, The University of Sydney, Sydney, NSW, Australia, where he researched electrostatic charge characteristics of inhalation medicine. He has a wide range of experience ranging from drug development and complex cell culture to microfabrication and high content imaging. He is a Co-Founder and the Chief Technology Officer at Mimetas B.V.-The Organ-on-a-Chip Company, Leiden, which markets and further develops the OrganoPlate. His research was published in over 20 peerreviewed papers and patents.

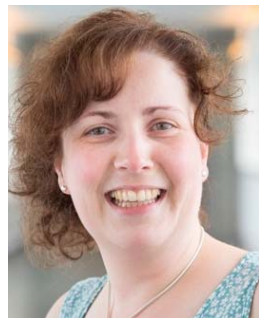

Klazina Kooiman (M'12) received the M.Sc. degree (cum laude) in biopharmaceutical sciences specializing in pharmaceutical technology from Leiden University, Leiden, The Netherlands, and the Ph.D. degree in ultrasound contrast agents for therapy from the Department of Biomedical Engineering, Thorax Center, Erasmus MC, Rotterdam, The Netherlands, in 2011.

She received the ICIN Fellowship in 2012, which enabled her to perform research for nine months at the Laboratory of Prof. F. S. Villanueva, Center for Ultrasound Molecular Imaging and Therapy, University of Pittsburgh Medical Center, Pittsburgh, PA, USA, from 2013 to 2014. In 2014, she acquired the prestigious VENI Grant from the Dutch Technology Foundation STW, which is a part of The Netherlands Organization for Scientific Research and the Erasmus MC Fellowship in 2015. She is currently an Assistant Professor and the Head of the Therapeutic Ultrasound Contrast Agent Group, Department of Biomedical Engineering, Thorax Centre, Erasmus MC, focusing on using ultrasound contrast agents for drug delivery and molecular imaging.

Dr. Kooiman was a recipient of the EFUSMB 2011 Young Investigator Award, Vienna, Austria. She is the Organizer of the Annual European Symposium on Ultrasound Contrast Imaging, Rotterdam, which is attended by approximately 175 scientists from universities and industries all over the world. 